\title{
Enzyme-functionalized thin-cladding long-period fiber grating in transition mode at dispersion turning point for sugar-level and glucose detection
}

\author{
Abdulyezir A. Badmos \\ Qizhen Sun \\ Zhongyuan Sun \\ Junxi Zhang \\ Zhijun Yan \\ Petro Lutsyk \\ Alex Rozhin \\ Lin Zhang
}




\title{
Enzyme-functionalized thin-cladding long-period fiber grating in transition mode at dispersion turning point for sugar-level and glucose detection
}

\author{
Abdulyezir A. Badmos, ${ }^{\mathrm{a}}$ Qizhen Sun, ${ }^{\mathrm{b}}$ Zhongyuan Sun, ${ }^{\mathrm{a}}$ Junxi Zhang, ${ }^{\mathrm{a}}$ Zhijun Yan, ${ }^{\mathrm{b}}$ Petro Lutsyk, ${ }^{\mathrm{a}}$ \\ Alex Rozhin, ${ }^{a}$ and Lin Zhang ${ }^{a, *}$ \\ ${ }^{a}$ Aston University, Aston Institute of Photonic Technologies, Birmingham, United Kingdom \\ ${ }^{b}$ Huazhong University of Science and Technology, School of Optical and Electronic Information and National Engineering Laboratory for Next \\ Generation Internet Access System, Wuhan, China
}

\begin{abstract}
Enzyme-functionalized dual-peak long-period fiber grating (LPFG) inscribed in 80- $\mu \mathrm{m}$-cladding B/Ge codoped single-mode fiber is presented for sugar-level and specific glucose detection. Before enzyme functionalization, the dual-peak LPFG was employed for refractive index sensing and sugar-level detection and high sensitivities of $\sim 4298.20 \mathrm{~nm} /$ RIU and $4.6696 \mathrm{~nm} / \%$ were obtained, respectively. Glucose detection probe was attained by surface functionalization of the dual-peak LPFG via covalent binding with aminopropyl triethoxysilane used as a binding site. Optical micrographs confirmed the presence of enzyme. The surface-functionalized dual-peak LPFG was tested with D-(+)-glucose solution of different concentrations. While the peak 2 at the longer wavelength was suitable only to measure lower glucose concentration $(0.1$ to $1.6 \mathrm{mg} / \mathrm{ml})$ recording a high sensitivity of $12.21 \pm 0.19 \mathrm{~nm} /(\mathrm{mg} / \mathrm{ml})$, the peak 1 at the shorter wavelength was able to measure a wider range of glucose concentrations $(0.1$ to $3.2 \mathrm{mg} / \mathrm{ml})$ exhibiting a maximum resonance wavelength shift of $7.12 \pm 0.12 \mathrm{~nm} / \mathrm{mg} / \mathrm{ml}$. The enzyme-functionalized dual-peak LPFG has the advantage of direct inscription of highly sensitive grating structures in thin-cladding fibre without etching, and most significantly, its sensitivity improvement of approximately one order of magnitude higher than previously reported LPFG and excessively tilted fibre grating (Ex-TFG) for glucose detection. $\odot$ The Authors. Published by SPIE under a Creative Commons Attribution 3.0 Unported License. Distribution or reproduction of this work in whole or in part requires full attribution of the original publication, including its DOI. [DOI: 10.1117/1.JBO.22.2.027003]
\end{abstract}

Keywords: dual-peak long-period fiber grating; D-(+)-glucose; covalent binding; micrograph; enzyme; wavelength shift.

Paper 160706RR received Oct. 13, 2016; accepted for publication Jan. 10, 2017; published online Feb. 6, 2017.

\section{Introduction}

Glucose, being the pivotal nutriment for human brain, muscles, and other body parts, essentially requires monitoring for any level imbalance to prevent malfunction and ensure healthy brain performance. Recent research findings have reported optical fiber-based biosensors with targeted applications in food safety, ${ }^{1}$ medical diagnosis, ${ }^{2,3}$ and environmental monitoring. ${ }^{4-6}$ Long-period fiber grating (LPFG) having the fundamental core mode coupled to the forward propagating cladding modes enables interaction between the propagating light and the ambient medium. This attribute allows LPFG to be utilized as a biosensor for direct probing of surrounding medium.

LPFG sensors have been reported to produce high-sensitivity resonant wavelength shift with the variation in refractive index (RI) of ambient medium, thereby offering label-free detection. Prominent citations on applications of LPFG-based biosensors include: antibody-antigen detection, ${ }^{7}$ chemical sensing, ${ }^{8} \mathrm{pH}$ sensing, ${ }^{9}$ glucose detection, ${ }^{10} \mathrm{DNA},{ }^{11,12}$ bacterial detection, and other medical diagnostic applications. ${ }^{13,14}$ Functionalizing the LPFG surface with molecular recognition elements such as enzymes, nucleic acids (DNA), antibodies, and antigens involves several bioreceptor immobilization methods which include covalent bonding, ${ }^{15,16}$ ionic bonding, ${ }^{17,18}$ absorption, ${ }^{19}$ avidinbiotin interaction, and cross-linking through a multifunctional

*Address all correspondence to: Lin Zhang, E-mail: I.zhang@aston.ac.uk reagent. ${ }^{20,21}$ While covalent bonding has been considered most effective of all the methods because it allows the active sites to remain chemically reactive and unobstructed, enzymes have also been considered usefully utilized as molecular recognition elements.

Dispersion turning point associated with dual-peak LPFG determines the condition of maximum sensitivity of cladding modes and occurs at longer wavelengths for lower order cladding modes with a shift to the shorter wavelength as the mode order increases. ${ }^{22}$ The high sensitivity exhibited by the dualpeak LPFG structure especially made in thin-cladding $(80-\mu \mathrm{m}$ diameter) fiber has prompted us to functionalize the dual-peak LPFG with enzyme for immunosensing of glucose. This serves as an alternative detection device to the prevalent electrochemical methods of glucose analysis. Here, we present an enzymefunctionalized, aqueous-dispersed, and actively targeted dualpeak LPFG with dispersion turning point in the near-infrared (NIR) region as a highly specific probe for sugar-level and glucose detection.

In previous reports, unmodified LPFGs have been used for nonselective analysis of very high-concentrated glucose solutions $(\geq 300 \mathrm{mg} / \mathrm{ml}),{ }^{23,24}$ but it offered insignificant changes in the RI and consequently no resonant wavelength shift. However, covalent immobilization methodology (conversion of glucose to gluconic acid) has been investigated for selective analysis of low-concentrated glucose solutions $(0.1$ to $3.0 \mathrm{mg} / \mathrm{ml})$ 10 and significant changes in the RI were obtained. This 
selective method was used in our research to show the correlation of the resonant wavelength shift of a dual-peak LPFG in a thin-cladding fiber to a range of $\mathrm{D}-(+)$-glucose concentrations.

\section{Materials and Methods}

\subsection{LPFG Inscription and Spectral Characteristics}

The prevalent point-by-point inscription method was used to fabricate dual-peak LPFGs of $300-\mu \mathrm{m}$ period on single-mode thin-cladding boron-germanium-doped fiber $(80-\mu \mathrm{m} \mathrm{B} / \mathrm{Ge}$ CA3596/877F/C-Nortel). An ultraviolet (UV) light source from a frequency-doubled argon $\left(\mathrm{Ar}^{+}\right)$laser at $244 \mathrm{~nm}$ (Sabre Fred, Coherent) was used for the LPFG inscription. The grating fabrication parameters include a period of $300 \mu \mathrm{m}$, grating length of $12 \mathrm{~mm}$, scanning speed of $0.2 \mathrm{~mm} / \mathrm{s}$, and laser power of $145 \mathrm{~mW}$. UV radiation propagating through the fiber core causes index modulation according to the phase matching condition: $:^{25}$

$\beta_{01}-\beta_{\text {cladding }}^{m}=\frac{2 \pi}{\Lambda}, \quad m=2,3,4,5, \ldots$

where $\beta_{01}$ and $\beta_{\text {cladding }}^{m}$ are the propagation constants of the fundamental core mode and the $m$ 'th-order copropagating cladding mode, respectively, and $\Lambda$ is the grating period. As a result of the parabolic characteristic of group index of higher-order cladding modes, a dispersion turning point was observed on the LPFG phase curve as $\left|\mathrm{d} \lambda_{\text {res }} / \mathrm{d} \Lambda\right| \rightarrow \infty$. For a given radius, the phase matching condition is met concurrently with one cladding mode in the positive dispersion region and the other in the negative dispersion region, thereby generating dual-peak resonances. ${ }^{22}$ We have theoretically simulated the cladding mode curves of the 80- $\mu$ m-diameter cladding fiber with $4.8-\mu \mathrm{m}$ core size for LPFGs with different periods and the results are plotted in Fig. 1(a). Due to the reduction in the fiber cladding diameter $(80 \mu \mathrm{m})$, the dispersion turning point has shifted to a lower order cladding mode curve region as compared with the normal $125-\mu \mathrm{m}$-cladding fiber. As per our calculation result shown in Fig. 1(a), the turning point for an LPFG

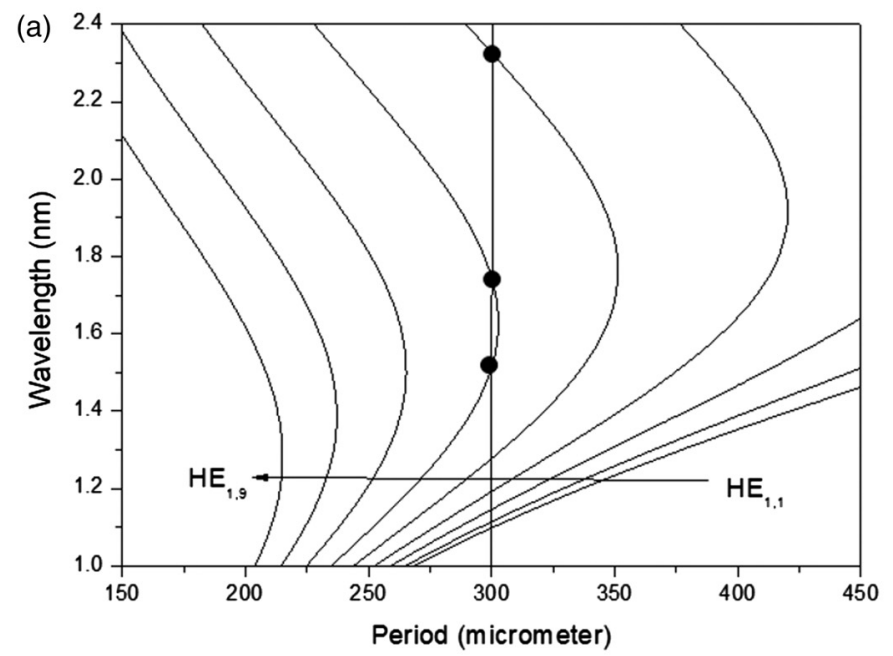

with $300-\mu \mathrm{m}$ period in $80-\mu \mathrm{m}$ thin-cladding fiber is associated with the cladding mode $\mathrm{HE}_{1,4}$.

According to the mode curves shown in Fig. 1(a), we see the paired attenuation bands of the dual-peak LPFG with a $300-\mu \mathrm{m}$ period in an $80-\mu$ m-cladding fiber would appear at the $\mathrm{HE}_{1,6}$ with wavelengths of 1520 and $1740 \mathrm{~nm}$. The real-time transmission spectrum evolution of the dual-peak LPFG with a $300-\mu \mathrm{m}$ grating period monitored on an optical spectrum analyzer (OSA AQ6375B-YOKOGAWA) as shown in Fig. 1(b) exhibits a dualpeak feature with the first of the dual attenuation peaks appearing at $1567.53 \mathrm{~nm}$ termed "peak 1" while the other peak at the longer wavelength, $\sim 1787.16 \mathrm{~nm}$, is termed "peak 2." Both resonance wavelengths are broadly in agreement with the simulated wavelengths of the dual peaks. These dual attenuation peaks are generated from the light coupling from the core mode to highsensitivity $\mathrm{HE}_{1,6}$ (dual peak) cladding mode, thereby interacting with the surrounding medium more responsively via evanescent fields. External perturbation effect on the evanescent fields induces changes in the effective index of the cladding mode, thus causing optically measurable wavelength shifts of the LPFG dual peaks according to the following equation: ${ }^{26}$

$\lambda_{m, \text { res }}=\left[n_{\text {eff,core }}^{01}\left(\lambda_{m}, n_{1}, n_{2}\right)-n_{\text {eff,clad }}^{0 m}\left(\lambda_{m}, n_{1}, n_{3}\right)\right] \Lambda$,

where $\lambda_{m \text {,res }}$ is the resonant wavelength due to coupling between fundamental core mode and the $m$ 'th cladding mode, $n_{\text {eff,core }}^{01}$ and $n_{\text {eff,clad }}^{0 m}$ are the effective indices of the fundamental core mode and the cladding mode, respectively, while the RIs of the core, cladding, and ambient medium are denoted by $n_{1}, n_{2}$, and $n_{3}$, respectively. It is clear from this mechanism that LPFGs are capable of detecting changes when a target bioanalyte interacts with a recognition element on their surfaces. ${ }^{12,27}$ In the evaluation, the response of the dual-peak LPFG to external perturbations was carried out: first, by characterizing the temperature sensitivity $\left(20^{\circ} \mathrm{C}\right.$ to $\left.80^{\circ} \mathrm{C}\right)$; then, by exposure to wide range of surrounding RI gels (1.305 to 1.444 RIU); sugar solutions of different concentrations ( $0 \%$ to $60 \%$ ), and finally, different glucose concentrations $(0.0$ to $3.2 \mathrm{mg} / \mathrm{ml})$ after the surface has been enzyme functionalized.

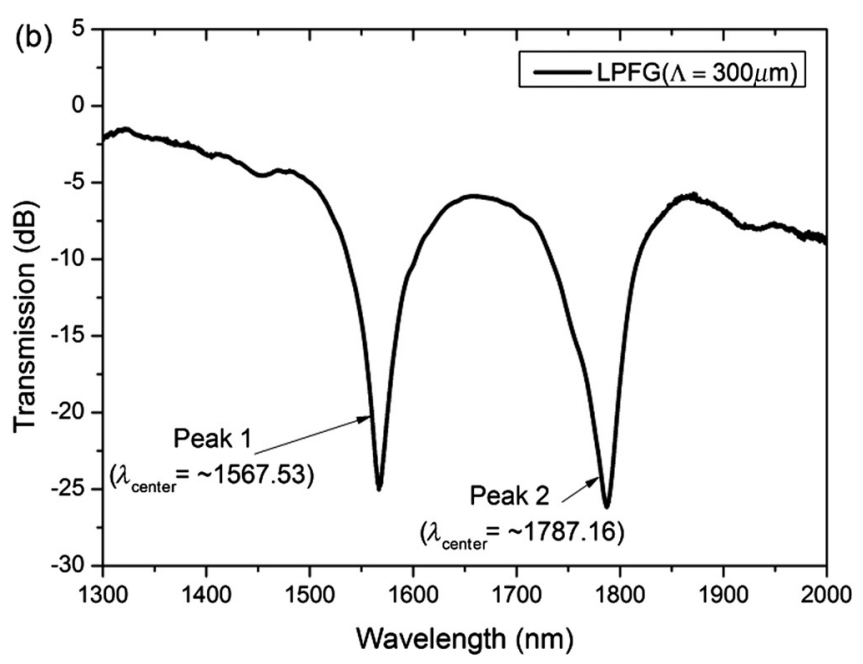

Fig. 1 (a) The simulated cladding mode curves of LPFGs of different periods in $80-\mu$ m-diameter cladding fiber with $4.8-\mu \mathrm{m}$ core size and (b) measured transmission spectrum of $80-\mu \mathrm{m}$-cladding LPFG showing dual peak in NIR region. 


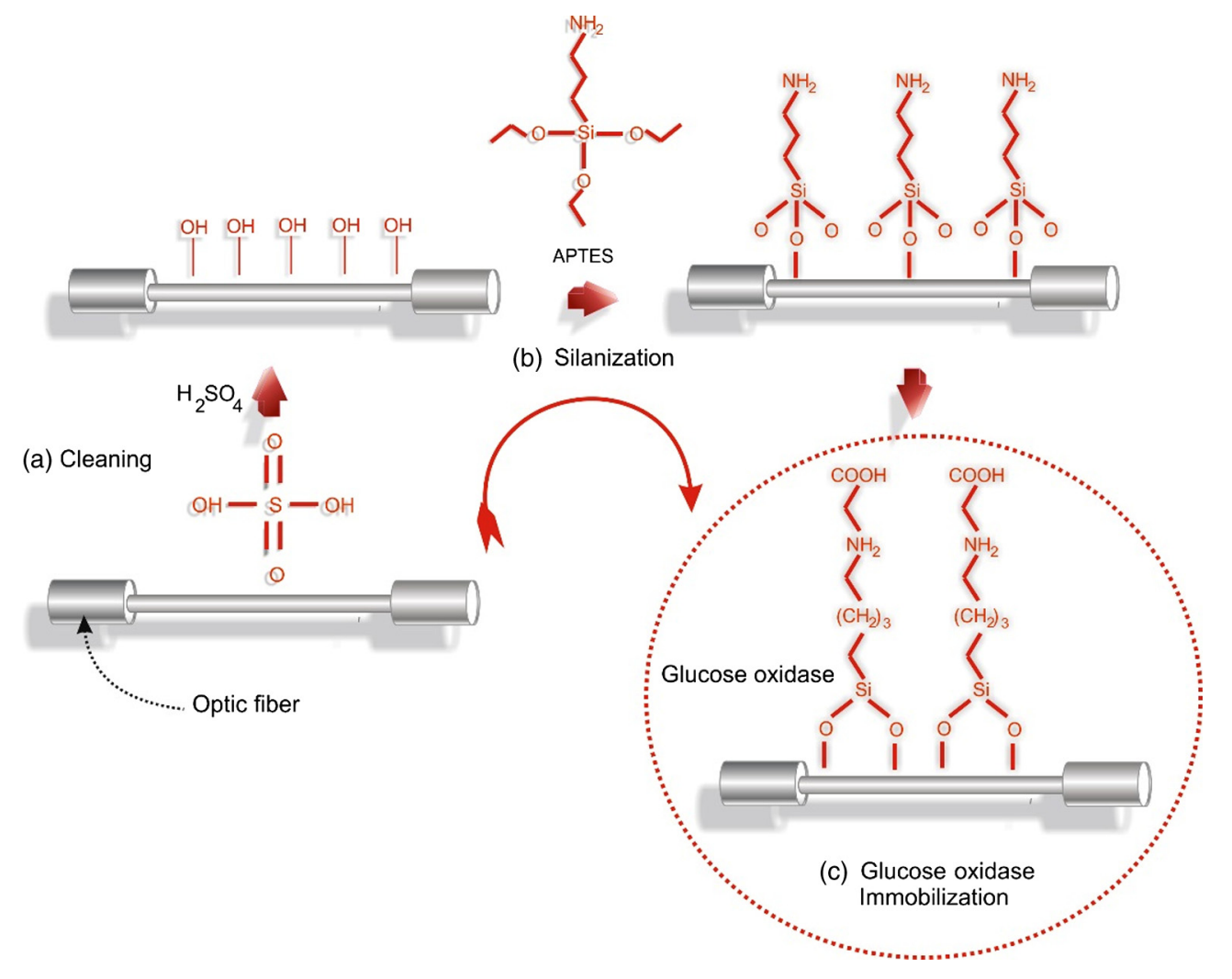

Fig. 2 Enzyme functionalization of LPFG surface: (a) cleaning process, (b) silanization process, and (c) glucose oxidase immobilization.

\subsection{Enzyme Functionalization of LPFG Surface}

The entire sample solutions were prepared by analytical-grade reagents and deionized water. All reagents used were purchased from Sigma Aldrich: sodium acetate (SA) buffer (form: liquid; S7899); D-(+)-glucose (form: powder; G7021); (3-aminopropyl) triethoxysilane (APTES) (form: liquid; A3648); and glucose oxidase (from Aspergillus niger) (form: buffered aqueous solution; G0543). The LPFG enzyme functionalization started with the cleaning process [Fig. 2(a)]. Here, the fiber was absolutely immersed in $\mathrm{HNO}_{3}$ solution $\left(5 \% \mathrm{v} / \mathrm{v} ; \sim 2 \mathrm{~h} ; 40^{\circ} \mathrm{C}\right)$ with a view to removing all contaminations, then washed thoroughly with deionized water and ethanol intermittently. Thereafter, the contamination-free fiber was immersed in $\mathrm{H}_{2} \mathrm{SO}_{4}$ solution $(95 \% \mathrm{v} / \mathrm{v}$ in $\mathrm{H}_{2} \mathrm{O}_{2} ; \sim 1 \mathrm{~h}$; room temperature) to activate the hydroxyl groups $(-\mathrm{OH})$ on the fiber surface. The fiber was left to dry under an incandescent lamp (40 W; $\left.240 \mathrm{~V} ; \sim 24 \mathrm{~h} ; \sim 40^{\circ} \mathrm{C}\right)$.

Afterward, it was submerged in (3-aminopropyl) triethoxysilane (APTES/ethanoic solution, 10\% v/v) which incubates the $-\mathrm{OH}$-activated fiber in the silanization process for $30 \mathrm{~min}$ at room temperature in order to covalently link the $\mathrm{NH}^{3+}$ groups of the APTES molecules to the - $\mathrm{OH}$ group on the fiber surface [Fig. 2(b)]. Note, the $\mathrm{NH}^{3+}$ group of the APTES molecules has been reportedly used to absorb biomolecules with negative charges like glucose oxidase and Escherichia coli bacteria. ${ }^{10,13}$ Then it was rinsed with deionized water and ethanol to remove noncovalently absorbed silane compounds.

Finally, glucose oxidase was immobilized on the $-\mathrm{NH}_{2}$ functionalized dual-peak LPFG surface [Fig. 2(c)] by immersion in $5 \mathrm{mg} / \mathrm{ml}$ buffered solution (SA) of glucose oxidase for $\sim 2 \mathrm{~h}$. During the incubation, glucose oxidase $-\mathrm{COOH}$ groups bonded with the $\mathrm{NH}^{3+}$ groups of the APTES molecules through covalent interaction. The enzyme-functionalized surface was later rinsed with SA buffer and left to dry in air. Each stage of the enzyme functionalization processes was examined under optical microscope (Zeiss Axioskop 2 mot plus upright Microscope) and the micrograph images taken are shown in Fig. 3.

The optical microscope transmission path (100 W halogenHAL 100) was used to observe the cleaned fiber, APTES-treated fiber, and glucose oxidase immobilized fiber [Figs. 3(a)-3(c)]. Optical recognition of the presence of glucose oxidase was as a result of its fluorescence property when observed under optical fluorescent microscopy (100×; ex. $479 \mathrm{~nm}$; light source: $200-\mathrm{W}$ metal halide arc lamp; 110 to $250 \mathrm{VAC} ; 2 \mathrm{~A} ; 50$ to $60 \mathrm{~Hz} ; 5^{\circ} \mathrm{C}$ to $30^{\circ} \mathrm{C}$-Lumen 200PRO) [Fig. 3(d)]. For better controlled measurement, the APTES-treated fiber was first observed under the fluorescent microscopy but no fluorescence property was detected; therefore, the fluorescence property is attributed only to the presence of glucose oxidase molecules. From the optical microscopic images obtained in Fig. 3, with each micrograph depicting each functionalization stage, it is evident that the surface of the fiber has been uniformly functionalized with the protocol used for the silanization and enzyme immobilization.

\subsection{Experimental Setup for Glucose Detection}

Figure 4 shows the experimental setup for the surrounding medium RI (SRI) sensing. The dual-peak LPFG fiber was mounted at each end on two three-dimensional translation stages (Thorlabs) with the grating region centralized above a test solution platform that was fixed to a micrometer stage (1 in., $25 \mathrm{~mm}$, Thorlabs) that is capable of vertical movement in either direction. Clean glass slides were placed intermittently on the 
Badmos et al.: Enzyme-functionalized thin-cladding long-period fiber grating in transition mode at dispersion turning point...

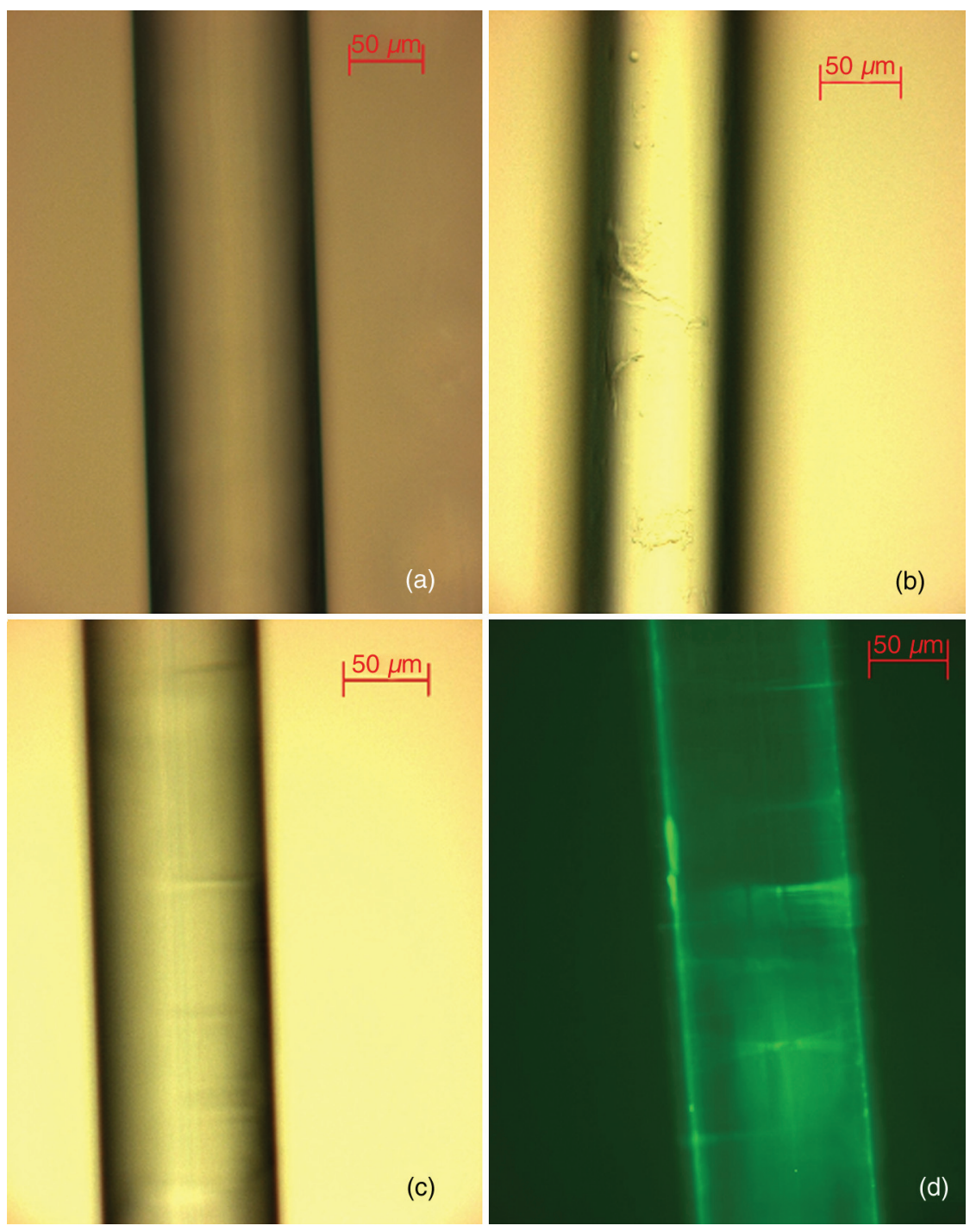

Fig. 3 Optical microscopic images of (a) cleaned optical fiber, (b) silanized optical fiber, (c) enzyme-functionalized optical fiber observed under transmitted light, and (d) enzyme's (fluorescent) presence on optical fiber surface.

Optical Spectrum Analyzer

YOKOGAWA AQ6375B

(1200nm - 2400nm range)

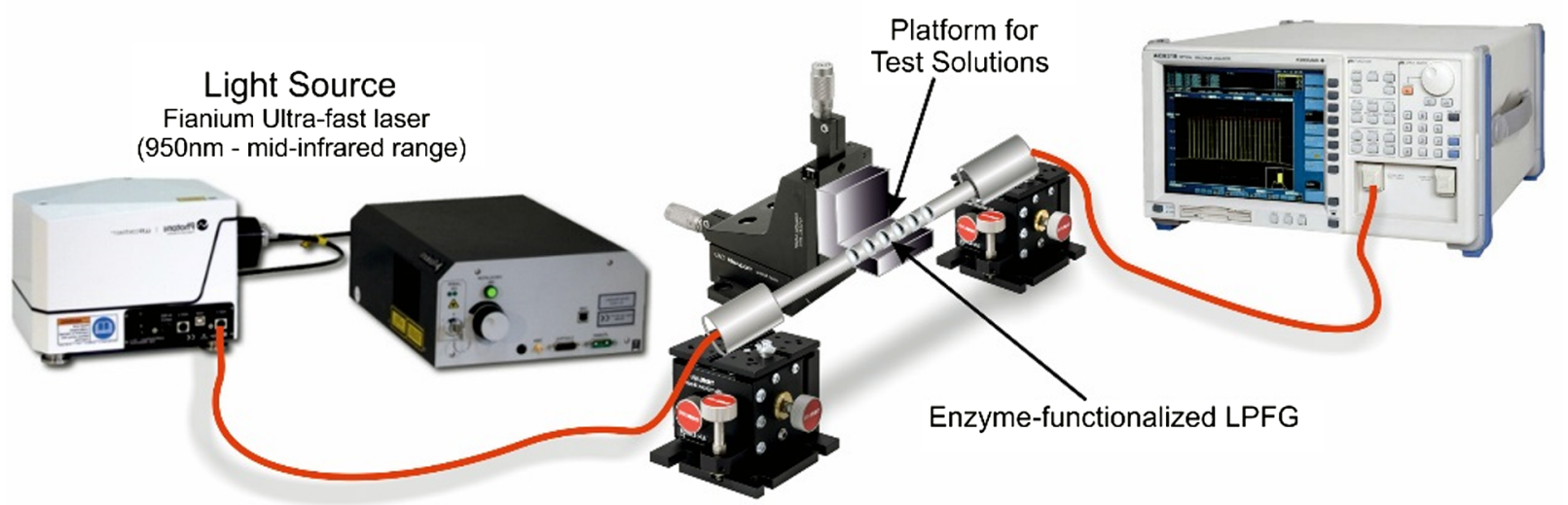

Fig. 4 Experimental setup of enzyme-functionalized LPFG biosensor for glucose detection. 
platform as each solution of different RIs was applied. One end of the fiber was connected to a supercontinuum light source (950 nm-mid-IR; Fianium) and the other end to the input port of the OSA. When light was transmitted through the fiber while the grating region was exposed in air, spectral measurement was taken on the OSA and recorded as reference. Subsequent submergence of the fiber in different solutions caused wavelength shift with respect to the reference as observed on the OSA and all transmission spectra were recorded.

The experimental setup for sensing all the test solutions (index gel, sugar solution, and glucose solution) is the same as Fig. 4 while that of the temperature measurement is a mere replacement of the micrometer stage by a Peltier connected to a temperature controller (ILX Lightwave). For the thermal measurement, supercontinuum light was transmitted through the fiber while the dual-peak LPFG is mounted on the Peltier. The temperature range of $20^{\circ} \mathrm{C}$ to $80^{\circ} \mathrm{C}$ in steps of $10^{\circ} \mathrm{C}$ was applied to the dual-peak LPFG and the transmission spectral response was measured using the OSA.

\section{Results and Discussion}

\subsection{Response to External Perturbations}

In order to optimize the performance of a biosensor, it is important to know all the constraints imposed by the sensing components. The dual-peak LPFG sensitivity to temperature was carried out to ascertain the level of cross-sensitivity of this sensor, which has been a major drawback for all LPFG sensors. Figure 5(a) plots the wavelength shift of the dual-peak LPFG in thin-cladding fiber against variation in temperature $\left(20^{\circ} \mathrm{C}\right.$ to $80^{\circ} \mathrm{C}$ ). As shown clearly in the figure, the dual peaks exhibit opposite movement with increasing temperature, which testifies the dual-peak feature as the coupled two cladding modes of the same order are situated very close to but on the opposite sites of the dispersion turning point. From the plots, we calculate the temperature sensitivities of the dual peaks: $-867.39 \pm 17.07 \mathrm{pm} /{ }^{\circ} \mathrm{C}$ and $676.03 \pm 26.95 \mathrm{pm} /{ }^{\circ} \mathrm{C}$ for peaks 1 and 2, respectively. In comparison with normal LPFGs, the typical temperature sensitivity of this dual-peak LPFG is relatively higher, which will impose a cross-sensitivity issue and must be taken into the consideration if the sensor is operated in an environment with fluctuation of thermal condition. Therefore, as the results of the temperature sensing measurement have shown that the dual-peak LPFG is prone to cross-sensitivity, the experiments for both sugar-level and glucose detection were strictly carried out under constant room temperature $\left(21 \pm 1^{\circ} \mathrm{C}\right)$.

However, there are situations in which several external perturbations coexist and the optical responses of LPFGs are generally a convolution of all these influences. Hence, to compensate for cross-sensitivity, an alternative is to either combine the LPFG structure with another grating structure (such as FBG) so as to facilitate simultaneous measurement of multiple parameters ${ }^{26}$ or modify the LPFG structure in order to be temperature insensitive such as LPFG in photonic crystal fiber ${ }^{28}$ or LPFG with rotary grooves carved by high-frequency $\mathrm{CO}_{2}$-laser pulses. $^{29}$

According to Eq. (2), the LPFG resonant wavelength depends on the effective index of the cladding mode, which in turn is dependent on the RI of the surrounding medium. Therefore, as the surrounding medium RI increases there is a corresponding shift in the resonant wavelength. The dualpeak LPFG was then first investigated as an RI sensor at a constant room temperature of $\sim 21^{\circ} \mathrm{C}$. To evaluate the RI sensitivity of the dual-peak LPFG, a series of index gels, 1.305 to 1.444 (Cargile), were employed to probe the response of the dualpeak LPFG to SRI variation. High SRI sensitivities were obtained, as shown in Fig. 5(b): peak 1 and peak 2 recorded sensitivities of -425.29 and $882.38 \mathrm{~nm} / \mathrm{RIU}$ in the RI range of 1.305 to 1.404 and -1991.10 and $4298.20 \mathrm{~nm} / \mathrm{RIU}$ in the RI range of 1.412 to 1.444 . It is interesting to note that both peaks show higher RI sensitivity in the second RI range, but the RI sensitivity of peak 2 is almost twice of that of peak 1 . This is a maximum recorded total-peak separation of $\sim 460 \mathrm{~nm}$ which is $23 \%$ improvement compared to $\sim 374 \mathrm{~nm}$ earlier reported for light cladding-etched dual-peak LPFG. ${ }^{30}$ Although, an RI sensitivity of $\sim 8734 \mathrm{~nm} / \mathrm{RIU},{ }^{31}$ which is much higher than ours, has been achieved on a thin-cladding dual-peak LPFG, the grating fiber was etched off from original $80 \mu \mathrm{m}$ to only $35 \mu \mathrm{m}$, undoubtedly reducing the mechanical strength of the grating and increasing its fragility, thus limiting its applicability.
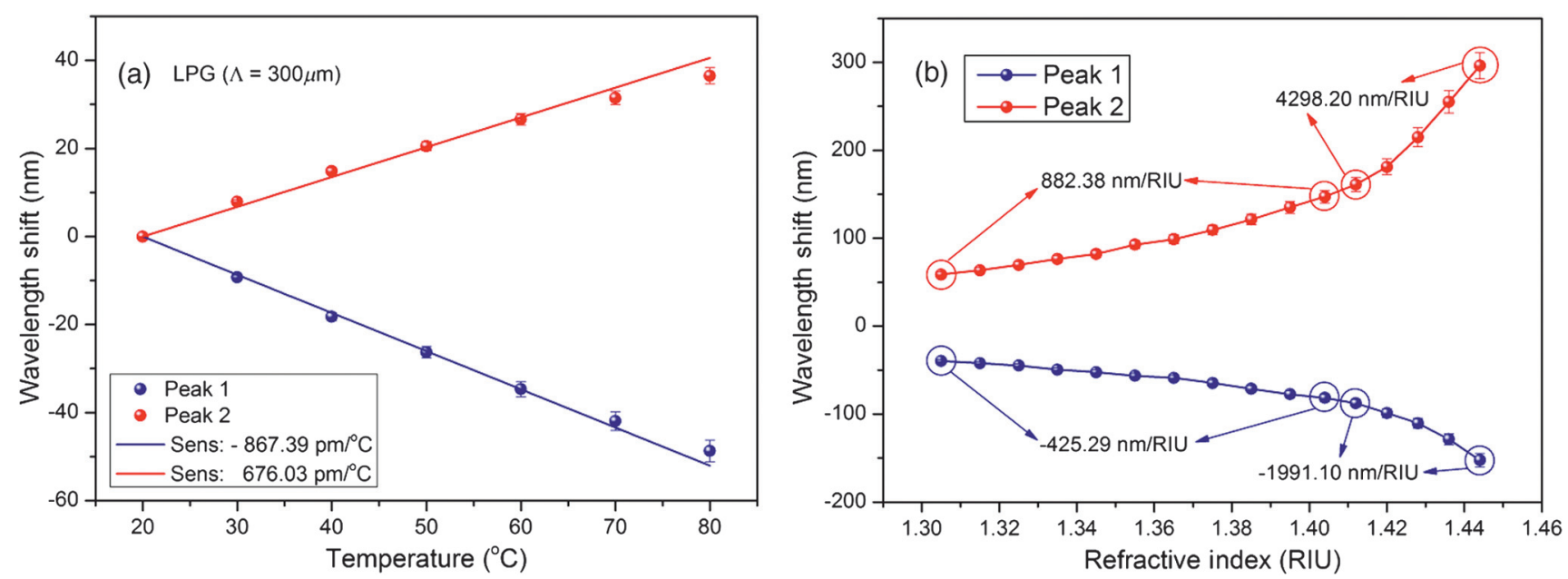

Fig. 5 (a) Plots of resonant wavelength variation against temperature; and (b) plots of resonant wavelength shift against surrounding medium RI variation of the $300-\mu$ m-period dual-peak LPFG in thin-cladding fiber. 

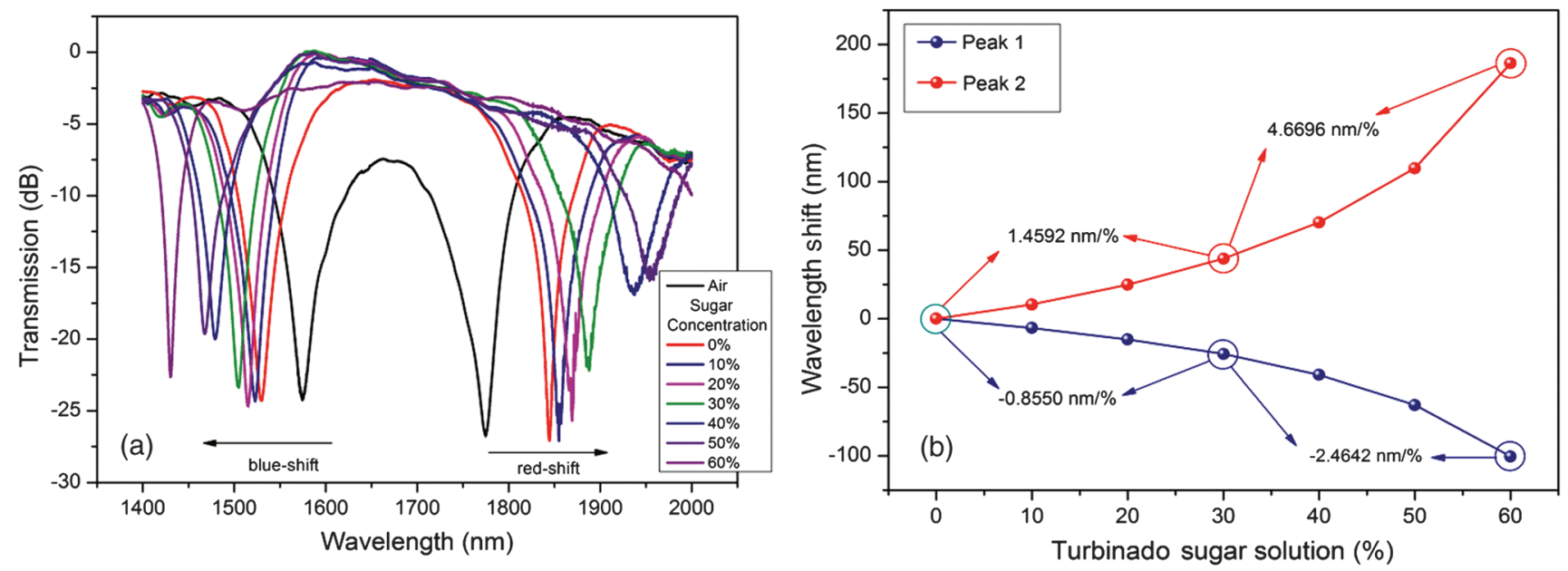

Fig. 6 (a) Transmission spectral evolution of $80-\mu$ m-cladding dual-peak LPFG to varying concentrations of turbinado sugar solution; and (b) plot of resonant wavelength shift of peak 1 and peak 2 against sugar solution.

Raw sugar (turbinado sugar) has been known to contain several useful nutrients (100 g of turbinado sugar contains$100 \mathrm{mg}$ potassium, $85 \mathrm{mg}$ calcium, $23 \mathrm{mg}$ magnesium, $3.9 \mathrm{mg}$ phosphorous, and $1.3 \mathrm{mg}$ iron) and less calories, and therefore is healthier than white sugar. ${ }^{32}$ Solutions of different concentrations $(0 \%$ to $60 \%)$ were made with the turbinado sugar and deionized water for sugar-level sensing using the same dualpeak LPFG at room temperature. Figure 6(a) shows the real-time shifting of the dual peaks in opposite directions with peak 1 shifting toward the shorter wavelength (blueshifting) while peak 2 drifts toward the longer wavelength (redshifting) as the concentration of the sugar solution increases. Both peaks recorded relatively high sensitivities to sugar-level detection with peak 1 showing a negative correlation coefficient of -0.8550 and $-2.4642 \mathrm{~nm} / \%$ for the sugar concentration levels of $0 \%$ to $30 \%$ and $30 \%$ to $60 \%$, respectively, while peak 2 shows stronger positive correlation coefficients of 1.4592 and $4.6696 \mathrm{~nm} / \%$ for the same two ranges [Fig. 6(b)]. From Fig. 6, it can be observed that peak 1 blueshifts by $\sim 100 \mathrm{~nm}$ while peak 2 redshifts by $\sim 200 \mathrm{~nm}$ for the entire sugar concentration ( $0 \%$ to $60 \%$ ) which are significantly higher than $\sim 19.8 \mathrm{~nm}$ peak redshift reported for concentration of hemoglobin in sugar solution ${ }^{30}$ as the sensitivity of our thin-cladding dual-peak LPFG has achieved over one order of magnitude improvement.

Since sucrose is sugar found naturally in fruits, vegetables, and sugar cane, monitoring of sugar level in industrial aqueous solutions can utilize this sensor for broader applications. The inclusion of the RI sensing and raw sugar solution detection experiments are specifically used to show the versatility of this sensor as not only being restricted to biosensing but also could find applications in food processing and environmental monitoring.

\subsection{Glucose Detection with Enzyme Functionalized Dual-Peak LPFG}

The enzyme functionalization of the dual-peak LPFG made it a biosensor for biomolecule detection. The specific enzyme (glucose oxidase) immobilized on the surface of the LPFG enables interaction with the surrounding glucose solution. Different concentrations of $\mathrm{D}-(+)$-glucose was prepared in SA buffer
(pH 5.2), which is a suitable reagent for this catalytic reaction. Equation (3) expresses the interaction of the glucose solution with glucose oxidase showing the conversion of $\mathrm{D}-(+)$-glucose to gluconic acid with concurrent release of hydrogen peroxide [1 unit of glucose oxidase (200 units $/ \mathrm{mg}$ ) oxidizes $1.0 \mu \mathrm{m}$ of $\mathrm{D}-(+)$-glucose to D-gluconic acid and $\mathrm{H}_{2} \mathrm{O}_{2}$ per minute at $\left.\mathrm{pH} \sim 5.1,35^{\circ} \mathrm{C}\right]$.

$$
\begin{aligned}
& \text { D- }(+) \text {-Glucose }+\mathrm{O}_{2} \\
& \quad+\mathrm{H}_{2} \mathrm{O} \stackrel{\text { Glucose oxidase }}{\longrightarrow} \text { D-Gluconic acid }+\mathrm{H}_{2} \mathrm{O}_{2} .
\end{aligned}
$$

This interaction causes change in the RI of the ambient medium and thus induces resonant wavelength shift in the transmission spectrum as observed on the OSA. After applying each glucose solution, deionized water was used to rinse the solution off the fiber surface to restore resonant peak back to the reference point and make ready for the next measurement of subsequent solutions. An average interaction time of $\sim 60 \mathrm{~s}$ was observed for each test solution (glucose solution). It was also observed that after enzyme functionalization of the LPFG surface, the dual-peak shifted around $\sim 15 \mathrm{~nm}$ from the initial position in air. Peak 1 was observed at around $\sim 1565 \mathrm{~nm}$ and peak 2 around $\sim 1802 \mathrm{~nm}$ as against initial positions. Figure 7(a) shows the transmission spectral evolution of peak 1 of the dual-peak LPFG in glucose solutions of different concentrations, showing the resonant peak drifting away from the initial reference position toward the shorter wavelength.

The test solutions (SA buffer/glucose) cover the glucose concentration range 0.1 to $3.2 \mathrm{mg} / \mathrm{ml}$ [Fig. 7(a), inset]. The obtained transmission spectra (peak 1) shows $\sim 30 \mathrm{~nm}$ blueshift with respect to the varying glucose solutions. However, as peak 2 redshifted, the concentration range was limited to lower values of between 0.1 and $1.6 \mathrm{mg} / \mathrm{ml}$ to be measured as the longest wavelength the light source can emit at around $2 \mu \mathrm{m}$. As shown in Fig. 7(b), for the glucose concentration range 0.1 to $3.2 \mathrm{mg} / \mathrm{ml}$, peak 1 has exhibited a linear response with a sensitivity of $7.115 \pm 0.119 \mathrm{~nm} /(\mathrm{mg} / \mathrm{ml})$, while for the lower glucose concentration range 0.1 to $1.6 \mathrm{mg} / \mathrm{ml}$, peak 2 recorded a higher sensitivity of $12.211 \pm 0.189 \mathrm{~nm} /(\mathrm{mg} / \mathrm{ml})$. From the results, we observe that the enzyme-functionalized dual-peak 

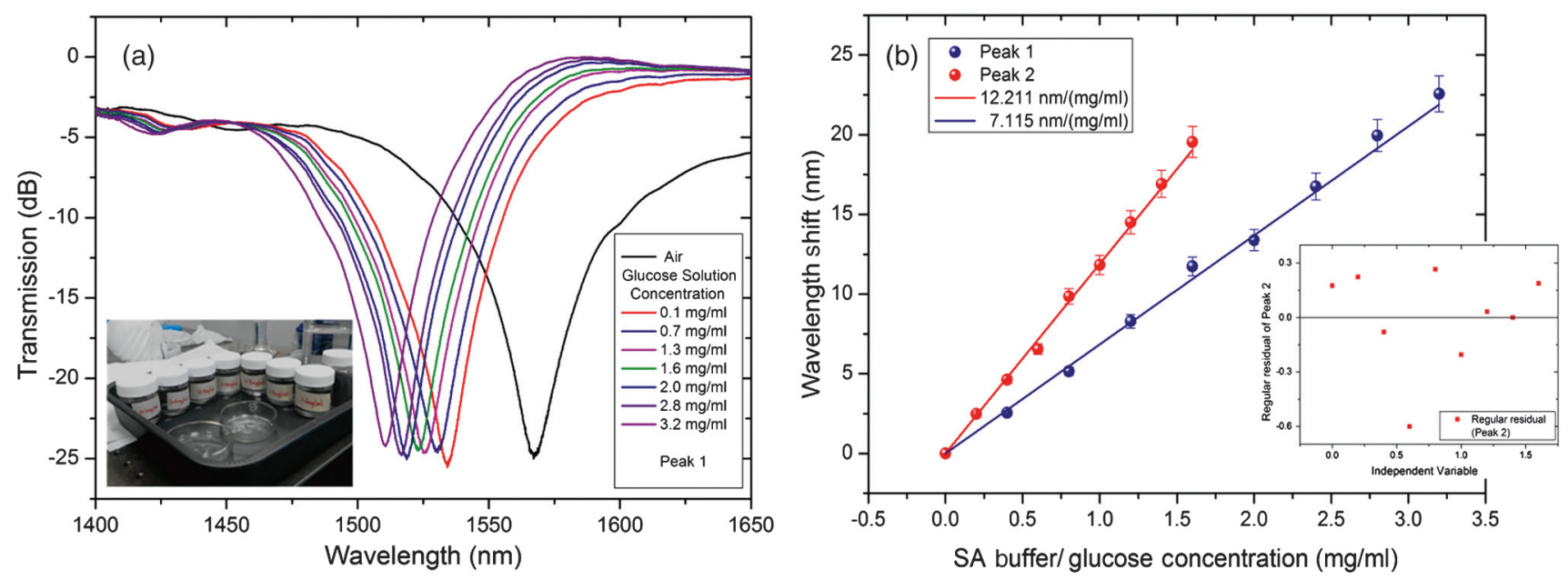

Fig. 7 (a) Transmission spectral evolution of peak 1 (of the dual-peak LPFG) to varying concentrations of SA buffer/D-(+)-glucose solution at different concentrations [inset: glucose solution $(0.1$ to $3.2 \mathrm{mg} / \mathrm{ml})$ ] and (b) plots of resonant wavelength shift of the dual-peak LPFG against glucose concentration (inset: regular residual of peak 2).

LPFG gives a linear response in the glucose concentration range ( 0.1 to $3.2 \mathrm{mg} / \mathrm{ml})$. In comparison with the previous reports, the sensitivity $\sim 7.115 \mathrm{~nm} /(\mathrm{mg} / \mathrm{ml})$ for peak 1 is approximately one order of magnitude higher than $\sim 0.806 \mathrm{~nm} /(\mathrm{mg} / \mathrm{ml})$ reported for normal $\mathrm{LPFG}^{10}$ and $\sim 0.298 \mathrm{~nm} /(\mathrm{mg} / \mathrm{ml})$ reported for $81^{\circ}-\mathrm{TFG}^{33}$ in similar glucose concentration range $(0.1$ to $3.2 \mathrm{mg} / \mathrm{ml}$ ).

In order to ascertain the RI cross-sensitivity in the glucose detection, an experiment was carried out using the bare dualpeak LPFG (without surface functionalization with glucose oxidase) in the same glucose concentration range (0.1 to $3.2 \mathrm{mg} / \mathrm{ml}$ ). The result shows that peak 1 recorded a sensitivity of $0.595 \mathrm{~nm} /(\mathrm{mg} / \mathrm{ml})$ that is $8.36 \%$ of the initial sensitivity of $7.115 \mathrm{~nm} /(\mathrm{mg} / \mathrm{ml})$ when the dual-peak LPFG was surfacefunctionalized with glucose oxidase. Therefore, the chemical interaction on the fiber surface between the glucose oxidase and the SA buffer/glucose solutions contributed the remaining $91.64 \%$ of the wavelength shift. In other words, the effect of RI cross-sensitivity to the glucose detection is $\sim 0.08$. The significantly improved sensitivity in comparison with earlier reports in Refs. 10 and 33 coupled with inherent high mechanical strength make this dual-peak LPFG sensor a viable and applicable alternative for supporting the instantaneous detection of the glucose concentration. Note, to ensure reproducibility of the result, the experiment was repeated three times with the glucose oxidase replenished for each repeated experiment, and the whole procedure shown in Fig. 2 was reiterated. The repeatability standard deviation of the experimental data was calculated to be 0.02 .

\section{Conclusion}

In summary, enzyme-functionalized dual-peak LPFG inscribed in $\mathrm{B} / \mathrm{Ge}$ codoped $80-\mu \mathrm{m}$-cladding single-mode fiber has been demonstrated and investigated for sugar-level and glucose concentration detection. Before the enzyme functionalization, the dual-peak LPFG has been investigated for RI sensing and a sensitivity of $4298.20 \mathrm{~nm} / \mathrm{RIU}$ has been achieved, which is a significant $23 \%$ improvement in total-peak separation as against an earlier report on light cladding-etched dual-peak LPFG. Also, sugar solution has been investigated for level concentration range $0 \%$ to $60 \%$, and a significant peak-wavelength shift of $\sim 200 \mathrm{~nm}$ has been achieved for the entire concentration range which is remarkably higher than $\sim 19.8 \mathrm{~nm}$ peak-wavelength shift earlier reported for concentration of hemoglobin in sugar solution. After the enzyme functionalization of the fiber surface, the dualpeak LPFG has been investigated for glucose concentration detection. The glucose detection has shown markedly high sensitivities of $12.211 \pm 0.189 \mathrm{~nm} /(\mathrm{mg} / \mathrm{ml})(0.1$ to $1.6 \mathrm{mg} / \mathrm{ml})$ for peak 2 and $7.115 \pm 0.119 \mathrm{~nm} /(\mathrm{mg} / \mathrm{ml})(0.1$ to $3.2 \mathrm{mg} / \mathrm{ml})$ for peak 1 which is one order of magnitude higher than earlier glucose detection results reported for normal LPFG and $81^{\circ}$-TFG. This dual-peak LPFG sensor, as a useful alternative, may find applications in food safety, medical diagnosis, and environmental monitoring.

\section{Disclosures}

We declare that there is no conflict of interest regarding this manuscript.

\section{Acknowledgments}

This work was supported by the Marie Curie International Incoming Fellowships (FP7-PEOPLE-2013-IIF, Grant Nos. 623473 and 913473). P.L. acknowledges support of EU FP 'Horizon-2020' Marie Skłodowska-Curie Individual Fellowship (FOC4SIP, \#654733).

\section{References}

1. M. D. P. T. Sotomayor et al., "Bi-enzyme optode detection system for oxalate determination based on a natural source of enzyme," Anal. Chim. Acta 447, 33-40 (2001).

2. C. DiCesare, I. Biran, and D. R. Walt, "Individual cell migration analysis using fibre-optic bundles," Anal. Bioanal. Chem. 382, 37-43 (2005).

3. D. Atias et al., "Chemiluminiscent optical fibre immunosensor for the detection of IgM antibody to dengue virus in humans," Sens. Actuators $B$ 140, 206-215 (2009).

4. V. G. Andreou and Y. D. Clonis, "A portable fibre-optic pesticide biosensor based on immobilized cholinesterase and sol-gel entrapped bromcresol purple for in-field use," Biosens. Bioelectron. 17, 61-69 (2002).

5. K. L. Brogan and D. R. Walt, "Optical fibre-based sensor: application to chemical biology," Curr. Opin. Chem. Biol. 9(5), 494-500 (2005). 
6. M. E. Bosch et al., "Recent development in optical fibre biosensors," Sensors 7(6), 797-859 (2007).

7. M. P. DeLisa et al., "Evanescent wave long-period fibre Bragg grating as an immobilized antibody biosensor," Anal. Chem. 72(13), 2895-2900 (2000).

8. J. A. Barnes et al., "Chemical sensing using a polymer coated longperiod fibre grating interrogated by ring-down spectroscopy," Sens. Actuators B 148(1), 221-226 (2010).

9. J. Goicoechea et al., "Optical fibre pH sensors based on layer-by-layer electrostatic self-assembled neutral red," Sens. Actuators B 132(1), 305311 (2008).

10. A. Deep et al., "Immobilization of enzyme on long period grating fibres for sensitive glucose detection," Biosens. Bioelectron. 33, 190-195 (2012).

11. H. M. R. Goncaives et al., "Biosensor for label-free DNA quantification based on functionalized LPGs," Biosens. Bioelectron. 84, 30-36 (2016).

12. X. Chen et al., "Real-time detection of DNA interactions with longperiod fibre-grating-based biosensor," Opt. Lett. 32, 2541-2543 (2007).

13. S. M. Tripathi et al., "Long period grating based biosensor for the detection of Escherichia coli bacteria," Biosens. Bioelectron. 35(1), 308-312 (2012).

14. C. L. Eggen et al., "Detection of lipid bilayer membranes formed on silica fibres by double-long period fibre grating laser refractometry," Sens. Actuators B 150(2), 734-741 (2010).

15. D. M. Disley et al., "Covalent coupling of immunoglobulin $\mathrm{G}$ to a poly (vinyl)alcohol-poly(acrylic acid) graft polymer as a method for fabricating the interfacial-recognition layer of a surface plasmon resonance immunosensor," Biosens. Bioelectron. 13(3-4), 383-396 (1998).

16. C. J. Stanford et al., "Covalent attachment of carbohydrate derivatives to an evanescent wave fiber Bragg grating biosensor," J. Sens. 2009, 982658 (2009).

17. C. Elosua et al., "Volatile alcoholic compounds fibre optic nanosensor," Sens. Actuators B 115(1), 444-449 (2006).

18. Z. Wang et al., "Biosensors employing ionic self-assembled multilayers adsorbed on long-period fibre gratings," Sens. Actuators B 139(2), 618 623 (2009).

19. X. Liu et al., "Molecular beacons for DNA biosensors with micrometer to submicrometer dimensions," Anal. Biochem. 283(1), 56-63 (2000).

20. R. Marks et al., "An innovative strategy for immobilization of receptor proteins on to an optical fibre by use of poly (pyrrole-biotin)," Anal. Biochem. 374(6), 1056-1063 (2002).

21. M. Lee and D. R. Walt, "A fibre-optic microarray biosensor using aptamers as receptors," Anal. Biochem. 282(1), 142-146 (2000).

22. X. Shu, L. Zhang, and I. Bennion, "Sensitivity characteristics of longperiod fibre gratings," J. Lightwave Technol. 20(2), 255-266 (2002).
23. D. W. Kim et al., "In-fibre reflection mode interferometer based on a long-period grating for external refractive-index measurement," Appl. Opt. 44(26), 5368-5373 (2005).

24. T. M. Libish et al., "Glucose concentration sensor based on long period grating fabricated from hydrogen loaded photosensitive fibre," Sens. Transducers J. 129(6), 142-148 (2011).

25. A. M. Vengsarkar et al., "Long-period fibre gratings as band-rejection filters," J. Lightwave Technol. 14(1), 58-65 (1996).

26. H. J. Patrick, A. Kersey, and F. Bucholtz, "Analysis of the response of long period fibre gratings to external index of refraction," J. Lightwave Technol. 16, 1606 (1998).

27. H. S. Jang et al., "Sensitive DNA biosensor based on a long-period grating formed on the side-polished fibre surface," Opt. Express 17, 38553860 (2009).

28. Y.-P. Wang et al., "Highly sensitive long-period fibre-grating strain sensor with low temperature sensitivity," Opt. Lett. 31(23), 3414-3416 (2006).

29. T. Zhu et al., "Highly sensitive temperature-independent strain sensor based on a long-period fibre grating with a $\mathrm{CO}_{2}$-laser engraved rotary structure," IEEE Photonics Technol. Lett. 21(8), 543-545 (2009).

30. X. Chen et al., "Dual-peak long-period fibre gratings with enhanced refractive index sensitivity by finely tailored mode dispersion that uses the light cladding etching technique," Appl. Opt. 46(4), 451455 (2007).

31. I. D. Villar et al., "Sensitivity optimization with cladding-etched long period fibre gratings at dispersion turning point," Opt. Express 24(16), 17680-17685 (2016).

32. M. A. Godshall and A. J. DeLucca, "Acetic acid, a major volatile constituent of brown sugar: its origin and measurement," J. Agric. Food Chem. 32(2), 390-393 (1984).

33. B. Luo et al., "Novel glucose sensor based on enzyme immobilized $81^{\circ}$ tilted fibre grating," Opt. Express 22(25), 30571-30578 (2014).

Abdulyezir A. Badmos received his BSc and MSc degrees in electrical/electronic engineering from the University of Lagos, Nigeria, in 2005 and 2008, respectively. He joined Aston Institute of Photonics Technologies, Aston University, Birmingham, UK, in April 2014 as a research student. His $\mathrm{PhD}$ research area includes optical fiber grating fabrication and nanocharacterization of in-fiber grating for biosensing applications. His research topic centers on "Advance optical fiber gratings for nanostructural characterization and biosensing applications." $\mathrm{He}$ is a member of SPIE.

Biographies for the other authors are not available. 